\title{
Lactate-upregulation of lactate oxidation complex-related genes is blunted in left ventricle of myocardial infarcted rats
}

\author{
D. Gabriel-Costa ${ }^{1,2}$, T.F. Cunha ${ }^{2}$, N.A. Paixão² ${ }^{2}$ R.S. Fortunato ${ }^{3}$, I.C.C. Rego-Monteiro ${ }^{3}$, \\ M.L.M. Barreto-Chaves ${ }^{4}$ and P.C. Brum ${ }^{2}$ \\ ${ }^{1}$ Programa de Pós-Graduação em Desempenho Humano Operacional, Universidade da Força Aérea, Rio de Janeiro, RJ, Brasil \\ ${ }^{2}$ Departamento de Biodinâmica do Movimento do Corpo Humano, Escola de Educação Física e Esporte, \\ Universidade de São Paulo, São Paulo, SP, Brasil \\ ${ }^{3}$ Instituto de Biofísica Carlos Chagas Filho, Universidade Federal do Rio de Janeiro, Rio de Janeiro, RJ, Brasil \\ ${ }^{4}$ Departamento de Anatomia, Instituto de Ciências Biomédicas, Universidade de São Paulo, São Paulo, SP, Brasil
}

\begin{abstract}
Lactate modulates the expression of lactate oxidation complex (LOC)-related genes and cardiac blood flow under physiological conditions, but its modulatory role remains to be elucidated regarding pathological cardiac stress. The present study evaluated the effect of lactate on LOC-related genes expression and hemodynamics of hearts submitted to myocardial infarction (MI). Four weeks after Ml or sham operation, isolated hearts of male Wistar rats were perfused for 60 min with $\mathrm{Na}^{+}$-lactate $(20 \mathrm{mM})$. As expected, Ml reduced cardiac contractility and relaxation with no changes in perfusion. The impaired cardiac hemodynamics were associated with increased reactive oxygen species (ROS) levels (Sham: $19.3 \pm 0.5$ vs MI: $23.8 \pm 0.3 \mu$ M), NADPH oxidase (NOX) activity (Sham: $42.2 \pm 1.3 \mathrm{vs} \mathrm{Ml:} 60.5 \pm 1.5 \mathrm{nmol} \cdot \mathrm{h}^{-1} \cdot \mathrm{mg}^{-1}$ ) and monocarboxylate transporter 1 (mct1) mRNA levels (Sham: $1.0 \pm 0.06$ vs Ml: $1.7 \pm 0.2$ a.u.), but no changes in superoxide dismutase (SOD), catalase, NADH oxidase (NADox), and xanthine oxidase activities. Lactate perfusion in MI hearts had no additional effect on ROS levels, NADox, and NOX activity, however, it partially reduced mct1 mRNA expression (MI-Lactate $1.3 \pm 0.08$ a.u.). Interestingly, lactate significantly decreased SOD (MI-Lactate: $54.5 \pm 4.2 \mu \mathrm{mol} \cdot \mathrm{mg}^{-1} \cdot \mathrm{min}^{-1}$ ) and catalase (MI: $1.1 \pm 0.1 \mathrm{nmol} \cdot \mathrm{mg}^{-1} \cdot \mathrm{min}^{-1}$ ) activities in MI. Collectively, our data suggest that under pathological stress, lactate lacks its ability to modulate the expression of cardiac LOC-related genes and the perfused pressure in hearts submitted to chronic MI. Together, these data contribute to elucidate the mechanisms involved in the pathogenesis of heart failure induced by MI.
\end{abstract}

Key words: Lactate; Lactate oxidation complex; Myocardial infarction; Gene expression; Perfusion pressure; Oxidative stress

\section{Introduction}

Lactate is a metabolic intermediary compound that links energy metabolism to different organs and tissues (1). The heart is an important lactate consumer especially during physiological stress conditions such as exercise (2-4). We and others have demonstrated that lactate modulates the expression of lactate oxidation complex (LOC)-related genes in both cardiac and skeletal muscle cells, which drive its usage as substrate fuel $(5,6)$. Lactate modulation of LOC-related genes occurs through the activation of redox-sensitive signaling pathways since lactate increases reactive oxygen species (ROS) levels and nuclear expression of the nuclear factor erythroid 2-related factor 2 (NRF-2), a redox-sensitive transcription factor $(5,6)$. In fact, lactate modulates redox homeostasis in cardiac tissue by increasing the levels of reduced $\beta$-nicotinamide adenine dinucleotide
(NADH) mainly due to the conversion of lactate to pyruvate by lactate dehydrogenase (LDH), which further activates a specific oxidase (NADH oxidase, NADox) leading to ROS production. Lactate also has an essential role in cardiac blood perfusion since it induces coronary artery relaxation. Montoya et al. (7) showed that it caused vasodilation in isolated aortic rings and coronary arteries in a nitric oxide (NO)-dependent manner. We have also observed that lactate perfusion reduces cardiac perfusion pressure in healthy isolated hearts (5).

Under pathological stress conditions, lactate exerts beneficial functions in energy synthesis. In heart failure (HF), both metabolic shifts of cardiac metabolism toward carbohydrate utilization and cardiac increase of lactate transport are adaptations that improve cardiac function, especially 
after myocardial ischemia (8). Many studies have demonstrated that oxidation of carbohydrates and other glycolytic substrates improve cardiac performance efficiency, resulting in enhanced heart contractility and less $\mathrm{O}_{2}$ utilization $(8,9)$. Taking into consideration data from healthy hearts, which demonstrate that lactate up-regulates the expression of LOC-related genes and increases coronary artery blood flux (5), we can hypothesize that it may also influence the establishment of HF. However, to our knowledge, no other study has previously investigated this hypothesis. The up-regulation of LOC-related gene expression and coronary artery vasodilation in hearts submitted to acute and chronic ischemia can be important compensatory mechanisms to prevent the decrease of cardiac performance and rescue the heart from further additional ischemic events, maybe delaying the onset of HF.

Considering the high indexes of mortality and the incidence of HF worldwide, especially from ischemic etiology $(10,11)$, it is extremely relevant to investigate the mechanisms related to the establishment of the disease, increasing the knowledge about its pathogenesis and further contributing to the development of new treatment strategies. Therefore, the aim of the present work was to investigate the effect of lactate perfusion in isolated hearts submitted to chronic experimental MI. We hypothesized that lactate would increase LOC-related gene expression and decrease cardiac perfusion pressure as observed previously in nonischemic hearts (5).

\section{Material and Methods}

\section{Animals}

Male Wistar rats weighing 250-300 g were used in the present study. Rats were placed in acrylic boxes lined with wood chips and access to water and standard chow ad libitum. Experiments were conducted according to the procedures stated in the Guide for the Care and Use of Laboratory Animals (National Institutes of Health) and approved by the Ethics and Research Committee of the Universidade de São Paulo (\#2011/55).

\section{Myocardial infarction surgery}

MI was obtained by ligation of the anterior descending coronary artery (ADCA). Animals were anesthetized with ketamine $(50 \mathrm{mg} / \mathrm{kg}, i p)$ and xylazine $(10 \mathrm{mg} / \mathrm{kg}, i p)$ and after thoracotomy, the ADCA was occluded preventing left ventricle (mainly anterolateral wall) blood supply. Some animals underwent the same procedures except the coronary artery ligation and were included in the sham-operated group. The day after surgery, echocardiographic analysis was conducted, and only infarcted animals that had lack of motility of the left ventricle anterior wall in $\mathrm{M}$ mode were included in the study. The animals were then assigned to three experimental groups: sham-operated perfused with Krebs-Henseleit $(\mathrm{KH})$ solution (Sham), MI perfused with $\mathrm{KH}$ solution (MI), and $\mathrm{MI}$ perfused with $\mathrm{KH}$ solution plus sodium lactate $(20 \mathrm{mM})$ (Ml-lactate).

\section{Isolated heart preparation}

After four weeks of the MI or sham surgery, the animals were euthanized and their hearts removed and placed on a Langendorff apparatus to test the effect of lactate perfusion as previously described by our group (5). Briefly, the isolated hearts were attached to a metal cannula in the Langendorff apparatus through the aorta for retrograde reperfusion. The flow was continuously kept throughout the experiment $( \pm 9 \mathrm{~mL} / \mathrm{min})$, and the hearts of Sham and $\mathrm{Ml}$ groups were perfused with $\mathrm{KH}$ solution of composition in: $118.0 \mathrm{mM} \mathrm{NaCl} ; 4.7 \mathrm{mM} \mathrm{KCl} ; 1.66 \mathrm{mM} \mathrm{MgSO}_{4}$; $1.18 \mathrm{mM} \mathrm{KH}_{2} \mathrm{PO}_{4} ; 1.5 \mathrm{mM} \mathrm{CaCl} ; 24.88 \mathrm{mM} \mathrm{NaHCO}_{3}$; $2.0 \mathrm{mM}$ glucose. The hearts of the Ml-lactate group were perfused with the same solution containing $20 \mathrm{mM}$ sodium lactate $(\mathrm{KH}$ plus lactate $=\mathrm{KHL})$. During the perfusion, the solutions were filtrated (Swinnex filter holder: $47 \mathrm{~mm}$, membrane pore: $0.22 \mu \mathrm{m}$; EMD Millipore, USA), bubbled with a carbogenic mixture $\left(95 \% \mathrm{CO}_{2}\right.$ and $\left.5 \% \mathrm{O}_{2}\right)$ and maintained at $37^{\circ} \mathrm{C}$ and $\mathrm{pH} \pm 7.4$. Subsequently, the left ventricle was accessed through the mitral valve, and a needle puncture was used to perforate the heart apex to avoid liquid accumulation. After that, a soft latex distensible balloon was placed into the left ventricle and inflated until diastolic pressure attained $\pm 10 \mathrm{mmHg}$. Both aortic and balloon cannulas were connected to pressure transducers and signals were amplified, digitalized, and stored for further analysis of the developed pressure (DP), heart rate $(\mathrm{HR})$, maximum positive and negative $\mathrm{dP} / \mathrm{dt}$ $\left(+\mathrm{dP} / \mathrm{dt}_{\max }\right.$ and $\left.-\mathrm{dP}_{\mathrm{d}} \mathrm{dt}_{\max }\right)$ and perfusion pressure (PP) (Power Lab-Lab Chart 7, ADInstruments, USA).

\section{Experimental protocol}

After the placement of the latex balloon into left ventricle, the hearts were equilibrated for $40 \mathrm{~min}$ beating spontaneously. $\mathrm{KH}$ or $\mathrm{KHL}$ were perfused for $60 \mathrm{~min}$ as previously described by Gabriel-Costa et al. (5). After 60 min, the left ventricle was dissected and readily frozen in liquid nitrogen and then at $-80^{\circ} \mathrm{C}$ for further analysis.

\section{Determination of lactate in cardiac homogenate and perfusate}

The lactate levels in the cardiac tissue and perfusate (collected at 0,30 , and $60 \mathrm{~min}$ ) were evaluated based on the technique of Rosenberg and Rush (12). Briefly, the tissues or perfusates were treated with perchloric acid $(3 \%, v / v)$, homogenized and centrifuged at $10,000 \mathrm{~g}$ for 20 $\min$ at $4^{\circ} \mathrm{C}$. The supernatant was used to measure lactate concentration. To determine lactate levels, the samples were first incubated with $0.2 \mathrm{M}$ of glycine-semicarbazide buffer and $0.02 \mathrm{M}$ of $\mathrm{NAD}^{+}$, and the absorbance was measured at a wavelength of $340 \mathrm{~nm}$ (R1). Then, $2 \mathrm{mg} / \mathrm{mL}$ of $\mathrm{LDH}$, obtained from rabbit muscle, was added in the preparation and incubated for $60 \mathrm{~min}$ at $40^{\circ} \mathrm{C}$. After that, 
a second reading was collected at the same wavelength (R2). $\mathrm{R} 1$ and R2 were used to calculate the net absorbance with and without $\mathrm{LDH}$, with the following formula $A=(R 2-$ $0.9 \mathrm{R} 1)-(\mathrm{B} 2-0.9 \mathrm{~B} 1)$, where $A$ is the net absorbance, $B 1$ is the blank absorbance without $L D H$, and $B 2$ is the blank absorbance with LDH. The concentration of lactate in $\mathrm{mM}$ was inferred by using the values of the net absorbance in a standard curve obtained between absorbance and increasing concentrations of lactate.

\section{Determination of NOX and NADox activities and reactive oxygen species levels}

NADox and nicotinamide adenine dinucleotide phosphate oxidase (NOX) activities were measured in enriched microsomal membrane (EMM) and enriched plasma membrane (EPM) of the perfused hearts, respectively. The EMM was obtained by homogenization of left ventricle tissue with phosphate buffer containing: $50.0 \mathrm{mM}$ sodium phosphate, $0.5 \mathrm{mM}$ dithiothreitol, $1.0 \mathrm{mM}$ ethylene glycolbis(2-aminoethyl ether)-N,N,N0,N0-tetraacetic acid, $250.0 \mathrm{mM}$ sucrose, $\mathrm{pH} 7.2$, and $5 \mu \mathrm{g} / \mathrm{mL}$ aprotinin, $34.8 \mu \mathrm{g} / \mathrm{mL}$ phenylmethanesulfonylfluoride (PMSF). The homogenate was centrifuged at $3,000 \mathrm{~g}$ for $15 \mathrm{~min}$ at $4^{\circ} \mathrm{C}$. The supernatant obtained was centrifuged for $30 \mathrm{~min}$ at $4^{\circ} \mathrm{C}$ at $100,000 \mathrm{~g}$ and the pellet resuspended in $1 \mathrm{~mL}$ of the same buffer. Another ultracentrifugation was obtained at the same conditions, and finally, the supernatant was resuspended in $1 \mathrm{~mL}$ of a phosphate buffer of composition: $50.0 \mathrm{mM}$ sodium phosphate, $1.0 \mathrm{mM}$ EGTA, $2.0 \mathrm{mM} \mathrm{MgCl}_{2}, 250.0 \mathrm{mM}$ sucrose, and $5 \mu \mathrm{g} / \mathrm{mL}$ aprotinin and $34.8 \mu \mathrm{g} / \mathrm{mL}$ PMSF, pH 7.2. The EPM was obtained by centrifugation of homogenized left ventricle tissue at $3,000 \mathrm{~g}$ for $15 \mathrm{~min}$ at $4^{\circ} \mathrm{C}$ and another subsequent centrifugation to obtain the pellet of the preparation (13).

Both NADox and NOX were measured under similar conditions. The samples were incubated in a medium containing phosphate buffer (150 mM), pH 7.2, Amplex Red $(50 \mu \mathrm{M})$, superoxide dismutase (SOD) $(100 \mathrm{U} / \mathrm{mL})$, and horseradish-peroxidase $(0.5 \mathrm{U} / \mathrm{mL})$. The reactions occurred with or without the corresponding substrates $\mathrm{NADH}$ or NADPH $(0.1 \mathrm{mM})$, respectively. To calculate the specific activity of both enzymes, the slope of the curve with the addition of NADH/NADPH was subtracted from the slope of the curves with addition of water for each sample. The fluorescence was detected at a wavelength of $563 \mathrm{~nm}$ to excitation and 587 to emission. A standard curve with known concentrations of $\mathrm{H}_{2} \mathrm{O}_{2}$ was used to transform the values to $\mathrm{nmol}$ or $\mu \mathrm{mol} \mathrm{H}_{2} \mathrm{O}_{2} \cdot \mathrm{min}^{-1} \cdot \mathrm{mg}^{-1}$. Since the preparations contained SOD, the results were referred as total $\mathrm{H}_{2} \mathrm{O}_{2}(5)$.

ROS generation was measured in EMM after reperfusion since NADox is present in microsomal fractions. The conditions used in this assay were similar to those employed in enzymatic measurements. The samples were incubated only with $50 \mu \mathrm{M}$ of Amplex Red, $100 \mathrm{U} / \mathrm{mL}$ of SOD, and $0.5 \mathrm{U} / \mathrm{mL}$ of horseradish-peroxidase (HRP) for $35 \mathrm{~min}$ at $30^{\circ} \mathrm{C}$.
After that, fluorescence levels at the end of the curve (Plato) were used to infer the ROS generation using a formula obtained in a standard curve with known concentrations of $\mathrm{H}_{2} \mathrm{O}_{2}$ and fluorescence transforming the values in $\mu \mathrm{M}$ of $\mathrm{H}_{2} \mathrm{O}_{2}$.

\section{Quantification of xanthine oxidase activity}

Xanthine oxidase (XO) activity was obtained as previously described by Veskoukis et al. (14). Samples were incubated with sodium potassium phosphate (33 mM, $\mathrm{pH} 7.5)$ and xanthine $(0.17 \mathrm{mM})$ and the reaction was immediately stopped with trichloroacetic acid. The samples were then centrifuged at $10,000 \mathrm{~g}$ for $15 \mathrm{~min}$ at $4^{\circ} \mathrm{C}$ and absorbance was read at $293 \mathrm{~nm}$. Subsequently, the same procedure was conducted after the incubation of the samples for $20 \mathrm{~min}$ at $37^{\circ} \mathrm{C}$. XO activity was obtained by subtracting the second from the first absorbance values. Calculation of $\mathrm{XO}$ activity was based on molar extinction coefficient of uric acid.

\section{Quantification of SOD and catalase activities}

SOD activity assay was based on the inhibition of xanthine/XO-driven cytochrome $\mathrm{C}$ reduction by SOD present in the sample. Left ventricle homogenates were obtained by macerating the tissue with potassium phosphate buffer containing: $50.0 \mathrm{mM} \mathrm{KH}_{2} \mathrm{PO}_{4}, 50.0 \mathrm{mM} \mathrm{K}_{2} \mathrm{HPO}_{4}, \mathrm{pH} 7.8$, followed by centrifugation at $10,000 \mathrm{~g}$ for $20 \mathrm{~min}$ at $4^{\circ} \mathrm{C}$. The rate of cytochrome $C$ reduction inhibition was measured in the absence or in the presence of the sample in a reaction medium containing: $1.18 \mathrm{mM}$ xanthine, $19.0 \mathrm{mM}$ cytochrome $\mathrm{C}$, and $\mathrm{XO}$, diluted in sodium phosphate buffer $(50 \mathrm{mM}, \mathrm{pH} 7.8)$ for $5 \mathrm{~min}$. SOD activity was calculated subtracting the rate of cytochrome $\mathrm{C}$ oxidase reduction inhibition in the presence and absence of the samples (15).

Catalase activity was measured as described by Weydert and Cullen (16). Muscle homogenates were obtained as described above. The rate of $\mathrm{H}_{2} \mathrm{O}_{2}$ decomposition by catalase was assessed by following the decay in absorbance at $240 \mathrm{~nm}$ for $4 \mathrm{~min}$ in the presence of $10 \mathrm{mM} \mathrm{H}_{2} \mathrm{O}_{2}$.

\section{RNA extraction and quantitative real-time RT-PCR}

Total RNA was isolated from left ventricle samples using Trizol (Invitrogen, USA). The RNA concentration and purity (260:280 $\mathrm{nm}$ ratios) were assessed in a spectrophotometer (Nanodrop 2000, Thermo Scientific, USA) and integrity was observed in an agarose gel $(2 \%$, w/v) electrophoresis. The cDNA was synthesized using Revertaid First Strand cDNA synthesis kit (Fermentas, USA). The genes analyzed were: $n r f-2$, monocarboxylate transporter (mct1), 4 (mct4), Idh, peroxisome proliferator receptor coactivator type 1 alpha $(p g c 1-\alpha)$, and cytochrome oxidase IV (coxiv) and cyclophilin were used as a reference genes. All primers were synthesized by Fermentas and their sequences are shown in Table 1. The amplifications were obtained using Maxima SYBR Green/ROX qPCR Master Mix (Fermentas) in ABI Prism 5700 Sequence 
Table 1. qRT-PCR primer sequences.

\begin{tabular}{|c|c|c|}
\hline Gene & Forward & Reverse \\
\hline$n r f-2$ & 5' GGCAGGAGCTATTTTCCATTCCCGAG 3' & 5' CTGGGGACAGTGGTAGTCTCAGCCTGC $3^{\prime}$ \\
\hline mct1 & 5' ACCGAGAGGGTCAGTGTTTG 3' & $5^{\prime}$ TGGAGGTAAGACTGCGTCAA 3' \\
\hline mct4 & 5' GGTCCCCTGGCTGCTATTAT 3' & $5^{\prime}$ TCCCATGGTCACACAAAGAA $3^{\prime}$ \\
\hline Idh & 5' GCAGCAGGGTTTCTATGGAG 3' & $5^{\prime}$ TGGAGACAGTGGGATTGTCA $3^{\prime}$ \\
\hline pgc1- $\alpha$ & $5^{\prime}$ GCGGACAGAACTGAGAGACC $3^{\prime}$ & $5^{\prime}$ CCATCATCCCGCAGATTTAC $3^{\prime}$ \\
\hline coxiv & $5^{\prime}$ GAACAAGGGCACCAATGAGT $3^{\prime}$ & 5' GTTGACCTTCATGTCCAGCA 3' \\
\hline Cyclophilin & $5^{\prime}$ TGGCAAGCATGTGGTCTTTGGGAAG $3^{\prime}$ & 5' GGTGATCTTCTTGCTGGTCTTGCCATTC $3^{\prime}$ \\
\hline
\end{tabular}

nrf2: Nuclear factor erythroid-2 related factor 2; mct1: monocarboxylate transporter 1; mct4: monocarboxylate transporter 4; Idh: lactate dehydrogenase; pgc1- $\alpha$ : peroxisome proliferator receptor coactivator type 1 alpha; coxiv: cytochrome oxidase IV.

Table 2. $\mathrm{pH}$ values of perfusate during 60 min of perfusion with $\mathrm{KH}$ or $\mathrm{KHL}$.

\begin{tabular}{lrrr}
\hline Cardiac perfusate & \multicolumn{1}{c}{0 min } & \multicolumn{1}{c}{$30 \mathrm{~min}$} & $60 \mathrm{~min}$ \\
\hline Sham & $7.39 \pm 0.1$ & $7.41 \pm 0.3$ & $7.45 \pm 0.1$ \\
Ml & $7.4 \pm 0.2$ & $7.5 \pm 0.1$ & $7.51 \pm 0.1$ \\
Ml-lactate & $7.45 \pm 0.1$ & $7.45 \pm 0.2$ & $7.5 \pm 0.2$ \\
\hline
\end{tabular}

KH: Krebs-Henseleit; KHL: Krebs-Henseleit+lactate (20 mM); MI: myocardial infarction. Data are reported as means \pm SE of 6 hearts.

Detection System (Applied Biosystems Inc., USA). Results are reported using the comparative cycle threshold $(\mathrm{Ct})$ method as described by the manufacturer. The $\Delta \Delta \mathrm{Ct}$ calculated from the subtraction of the $\Delta \mathrm{Ct}$ of the gene of the $\mathrm{Ml}$ and Ml-lactate groups ( $\Delta \mathrm{Ct}$ was calculated by subtraction of the gene Ct's from the reference-cyclophilin $\mathrm{Ct}$ ) from the $\Delta \mathrm{Ct}$ of the control group (Sham) are reported in $2^{-\Delta \Delta C t}$. Control group levels were arbitrarily set to 1 .

\section{Statistical analysis}

Data are reported as means \pm SE. The means were compared with non-paired $t$-test or one-way analysis of variance and Tukey's post hoc, when necessary. The results were considered significantly different when $\mathrm{P}<0.05$.

\section{Results}

Lactate levels in the perfusate were obtained at $30 \mathrm{~min}$ and $60 \mathrm{~min}$. No differences were observed between Sham and $\mathrm{Ml}$ groups $(2.1 \pm 0.2$ vs $1.8 \pm 0.1 \mathrm{mM}$, respectively) at $60 \mathrm{~min}$, but it increased significantly after lactate perfusion (Ml-lactate group: $22.3 \pm 0.8 \mathrm{mM}$ ). These values were kept constant throughout the experiment $(30,60,90 \mathrm{~min}$; data not shown). Cardiac lactate basal levels were not altered by MI $(1.0 \pm 0.9$ vs $1.5 \pm 1.0 \mathrm{mM}$, for Sham and Ml groups, respectively). However, lactate perfusion induced a significant increase in lactate levels when Ml-lactate hearts were compared to both Sham and MI hearts $(4.7 \pm 0.8 \mathrm{mM})$. We also tested whether lactate administration would acidify the isolated heart perfusate along the $60 \mathrm{~min}$ of lactate perfusion at $20 \mathrm{mM}$ (Table 2). As expected, there were no sign of acidosis in the perfusate throughout the experiment.

The cardiac hemodynamic parameters recorded during 60 min of perfusion of the hearts with $\mathrm{KH}$ or $\mathrm{KHL}$ solutions are shown in Figure 1. As expected, MI significantly reduced $\mathrm{DP}$ (Figure $1 \mathrm{~A}),+\mathrm{dP}^{\mathrm{d}} \mathrm{dt}_{\max }$ (Figure $1 \mathrm{~B}$ ), and $-\mathrm{dP} /$ $\mathrm{dt}_{\max }$ (Figure $1 \mathrm{C}$ ), and lactate perfusion had no impact on cardiac contractile function. Surprisingly, lactate perfusion did not change PP in the Ml-lactate group (Figure 1D). Additionally, HR (Figure 1E) was not altered by either lactate perfusion or MI.

Figure 2A shows that $\mathrm{Ml}$ induced a significant increase in cardiac ROS levels, which was not modified by lactate perfusion $(19.8 \pm 0.4$ vs $23.8 \pm 0.27$ and $24.3 \pm 1.3 \mu \mathrm{M}$, for Sham, MI, and MI-lactate groups, respectively). The increased cardiac ROS levels in both MI groups were associated to a high NOX activity in $\mathrm{Ml}$ groups (Figure 2B, $42.5 \pm 1.3$ vs $60.5 \pm 1.5$ and $61.1 \pm 1.8 \mathrm{nmol} \cdot \mathrm{h}^{-1} \cdot \mathrm{mg}^{-1}$, for Sham, MI, and MI-lactate groups, respectively), while NADox (Figure 2C) and XO (Figure 2D) activities remained similar among groups. Likewise, both SOD (Figure 2E) and catalase (Figure 2F) activities were not altered in MI hearts. Unexpectedly, the activity of both antioxidant enzymes was reduced by lactate perfusion in MI hearts (SOD: $76.5 \pm 4.1$ vs $72.0 \pm 9.0$ and $54.5 \pm 4.2 \mu \mathrm{mol} \cdot \mathrm{mg}^{-1}$. $\mathrm{min}^{-1}$ and catalase: $1.61 \pm 0.15$ vs $1.12 \pm 0.16$ and $1.08 \pm$ $0.10 \mathrm{nmol} \cdot \mathrm{mg}^{-1} \cdot \mathrm{min}^{-1}$, for Sham, Ml, and Ml-lactate groups, respectively). The reduced SOD and catalase activities in 

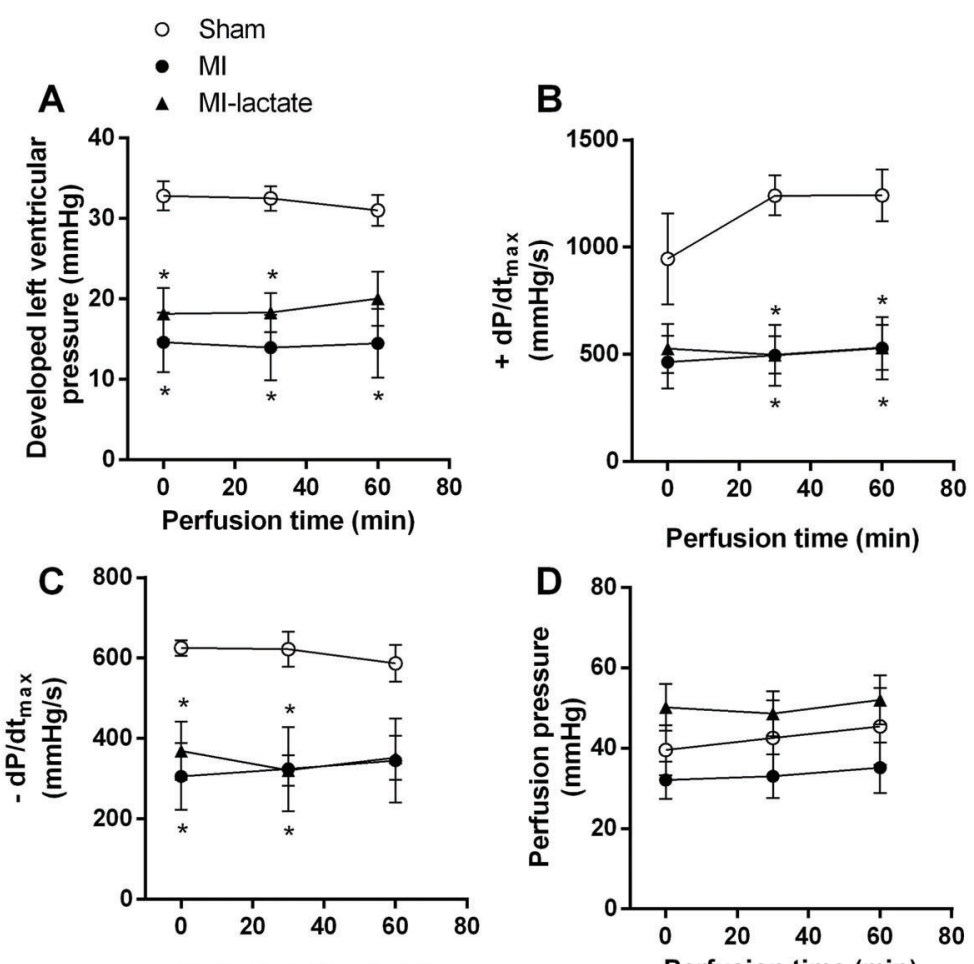

Perfusion time (min)

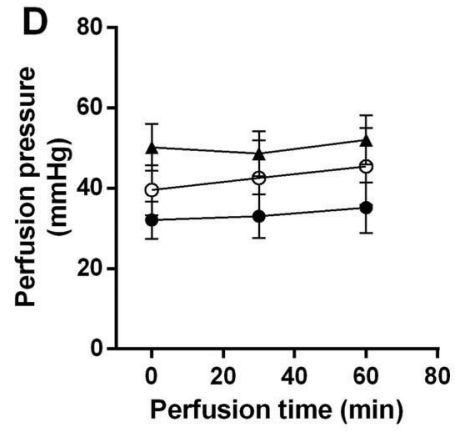

Figure 1. $A$, Left ventricular developed pressure, $B$, maximum positive $\left(+\mathrm{dP}^{\mathrm{d}} \mathrm{dt}_{\max }\right.$ and $C$, negative $\left(-\mathrm{dP}_{\mathrm{d}} \mathrm{dt}_{\max }\right), D$, perfusion pressure, and $E$, heart rate after 60 min of perfusion with KrebsHenseleit or $\mathrm{KH}$ plus lactate. Ml: myocardial infarction. Data are reported as means \pm SE of 6 hearts. ${ }^{*} P<0.05$ compared to Sham group (ANOVA).

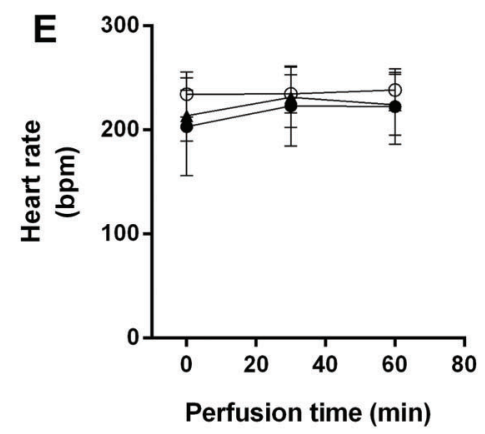

lactate-perfused MI hearts were not accompanied by changes in nrf-2 mRNA levels, a redox-sensitive transcription factor that regulates SOD and catalase expression (Figure 2G).

As lactate transportation through cell and mitochondria membranes in cardiac cells is mainly mediated by mct 1 and $m c t 4$, we have evaluated mct 1 and $m c t 4$ mRNA levels in isolated hearts. mct1 mRNA levels were significantly increased after $\mathrm{Ml}$, however, lactate perfusion reduced mct1 mRNA levels toward Sham group levels (Figure 3A, $1.0 \pm 0.05,1.6 \pm 0.22$, and $1.2 \pm 0.07$ a.u for Sham, MI, and Ml-lactate, respectively). mct4 mRNA levels were not modified by either $\mathrm{Ml}$ or Ml-lactate perfusion (Figure 3B). Likewise, no changes were observed in mRNA levels of other LOC-related genes among groups, such as Idh (Figure 3C), pgc1- $\alpha$ (Figure 3D), and coxiv (Figure 3E).

\section{Discussion}

The main finding of the present study is that lactate lacks its modulatory role in the expression of LOC-related genes and in the relaxation of coronary arteries in hearts submitted to chronic pathological stress, such as MI. In parallel, the infarcted hearts presented a sustained increase in ROS levels, and no changes in mct4, Idh, pgc1- $\alpha$, and coxiv mRNA levels were observed in either Ml or lactateperfused Ml groups. In contrast, the levels of mct 1 mRNA were partially reduced, and SOD and catalase activities decreased after lactate perfusion in $\mathrm{Ml}$ isolated hearts.

We have previously demonstrated in healthy isolated hearts that lactate induced a slight, but significant increase in ROS production that was mainly associated with NADox activity, nrf-2 nuclear expression, and LOC gene expression (5). 

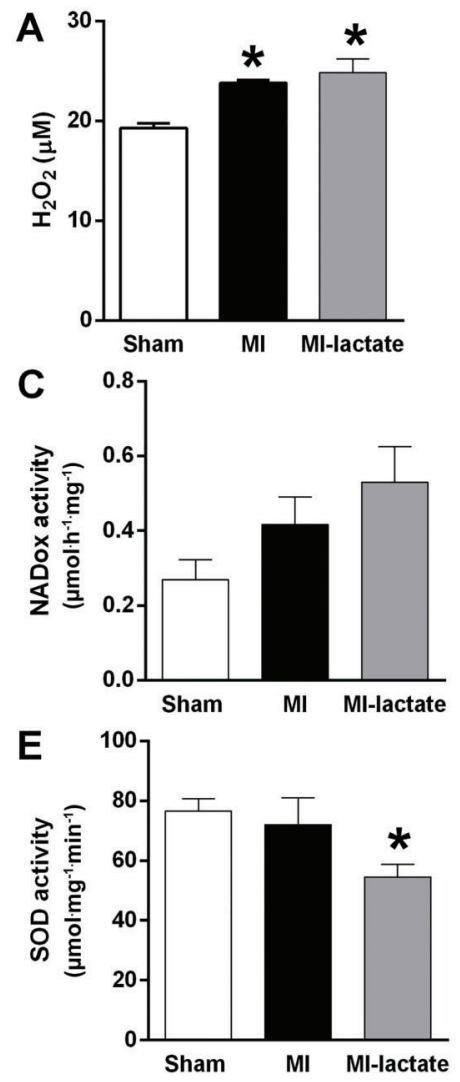
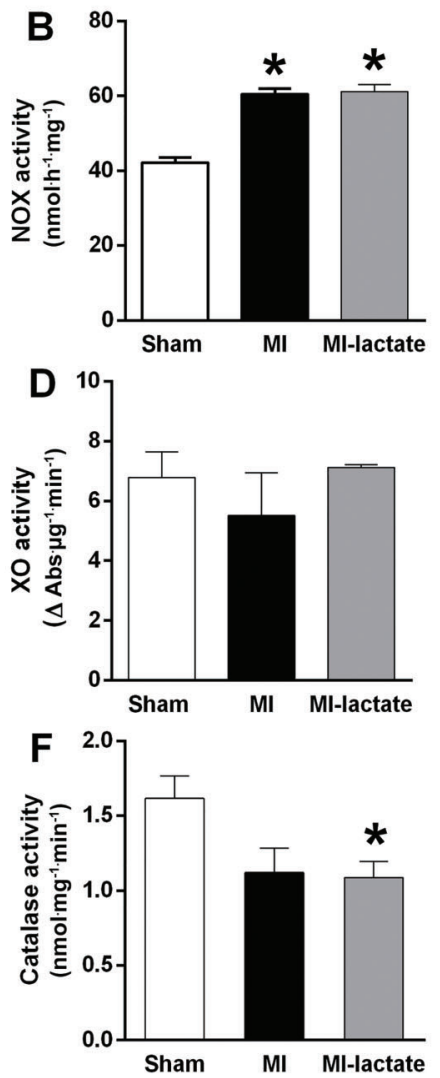

Figure 2. $A$, Reactive oxygen species $\left(\mathrm{O}_{2} \cdot \%\right.$ $\mathrm{H}_{2} \mathrm{O}_{2}$ ) and activity of $B$, nicotinamide adenine dinucleotide phosphate oxidase (NOX), $C, N A D H$ oxidase (NADox), $D$, xanthine oxidase (XO), $E$, superoxide dismutase (SOD), $F$, catalase, and $G$, total nuclear factor erythroid 2-related factor 2 (nrf-2) mRNA levels in hearts perfused for $60 \mathrm{~min}$ with Krebs-Henseleit $(\mathrm{KH})$ and $\mathrm{KH}$ plus lactate. MI: myocardial infarction. Data are reported as means \pm SE of 6 hearts. ${ }^{*} \mathrm{P}<0.05$ vs Sham group (ANOVA).

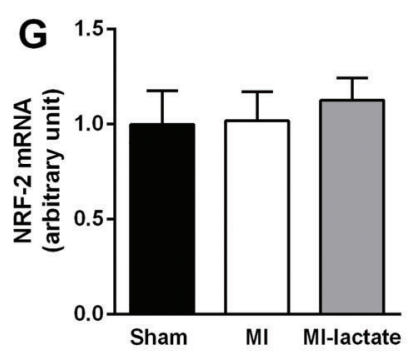

Presently, we demonstrated that this response was lacking in MI hearts, since we observed that $\mathrm{Ml}$ per se increased ROS levels $(20 \mu \mathrm{M})$ in cardiac cells with no further increment after lactate perfusion. In fact, the enhancement of ROS concentration was associated to NOX but not NADox activity. These data are in agreement with an established consensus, which states that Ml induces both sympathetic nervous and renin-angiotensin systems hyperactivity that recognizably up-regulate components of NOX complex and ROS levels in cardiac muscle (17-19). The lack of response to lactate perfusion after Ml suggests that an excessive prooxidant environment induced mainly by NOX activation somehow prevented the ability of lactate in modulating gene expression by redox-sensitive signaling pathways.

The lactate-induced pro-oxidant status resulted in responses other than up-regulation of LOC-related genes.
In fact, lactate partially reduced $m c t 1$ expression. Although the mechanisms involved in these effects were not investigated herein, we consider these findings relevant to HF treatment, as they contribute to elucidate the role of lactate in the establishment of the disease. Based on previous data of our group, we hypothesized that after chronic ischemia, lactate could contribute to enhancing energy metabolism by increasing the expression of proteins involved in its oxidation. It is reasonable to suggest that up-regulation of LOCrelated gene expression is a positive adaptation that may further contribute to delay HF onset. However, the present data do not support this hypothesis. Other interesting data obtained were that lactate perfusion in MI hearts decreased both catalase and SOD activities. From our knowledge, this is the first study suggesting that lactate negatively modulates antioxidant enzyme activities in MI hearts. The physiological 

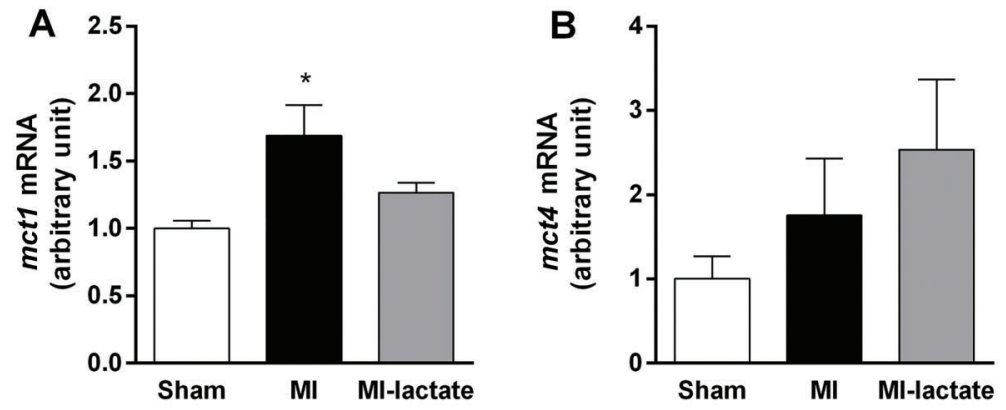

Figure 3. $A$, Total mRNA levels of monocarboxylate transporter $(m c t) 1, B, m c t 4, C$, lactate dehydrogenase $(I d h)$, and $D$, proliferator receptor coactivator type 1 alpha (pgc1- $\alpha)$ and E, cytochrome oxidase IV (coxiv) in left ventricle tissue after $60 \mathrm{~min}$ of perfusion with Krebs-Henseleit $(\mathrm{KH})$ and $\mathrm{KH}$ plus lactate. Ml: myocardial infarction. Data are reported as means \pm SE of 6 hearts. ${ }^{*} \mathrm{P}<0.05$ vs Sham group (ANOVA).
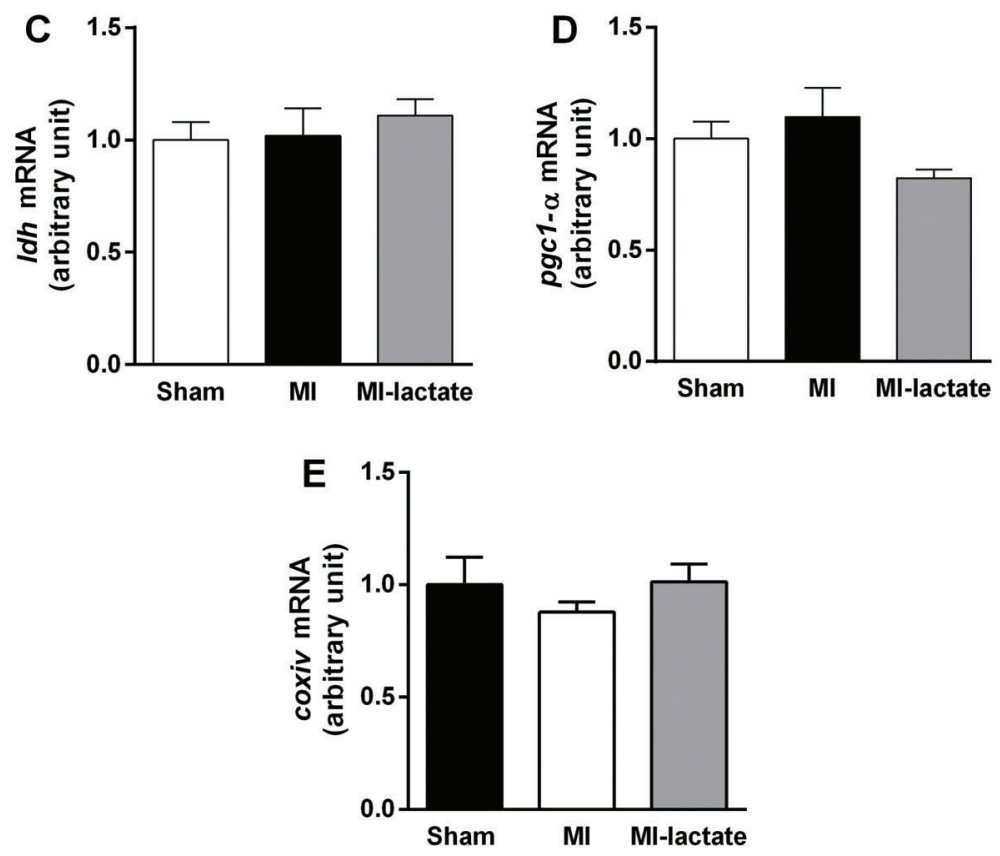

consequences of reduced antioxidant defense in HF are deleterious since oxidative stress is directly involved in its pathogenesis. Therefore, the results of the present study suggest that lactate might be one of the factors that contribute to the pathogenesis of HF.

As expected, Ml-induced cardiac dysfunction was demonstrated by reduced cardiac DP, and impaired cardiac inotropic $(+\mathrm{dP} / \mathrm{dt})$ and lusitropic $(-\mathrm{dP} / \mathrm{dt})$ function, which were not changed by lactate perfusion in isolated MI hearts. Interestingly, lactate perfusion in Ml hearts did not reduce cardiac perfusion pressure as we previously observed in isolated healthy hearts (5). We believe that it was in consequence of the Ml-induced endothelium dysfunction, since Montoya et al. (7) have previously demonstrated that lactate-induced vasodilation was endothelium-NO-dependent. Data obtained from lactate and $\mathrm{pH}$ levels, both in perfusate and cardiac tissue, suggest that the hearts were well-oxygenated and relying on aerobic metabolism. Our data corroborate the results of Opie (20), who used similar perfusion pressures.
Taken together, our data provide evidence that MI blunted the ability of lactate in modulating LOC-related gene expression and cardiac perfusion in isolated hearts. The practical implications of our findings are that lactate did not rescue cardiac function and hemodynamics in ischemic HF. Instead, it contributed to decrease mct1 mRNA expression and antioxidant enzymatic defense. Although increasing evidence suggests that switching metabolic fuel usage toward glycolytic fuel (e.g. lactate) oxidation increases cardiac function (8), our data provided evidence that it might not be helpful at all. In this sense, future studies should address the implication of the role of lactate in all phases of $\mathrm{HF}$ in cardiac gene expression and perfusion after MI.

\section{Acknowledgments}

D. Gabriel-Costa held a post-doctoral grant from Conselho Nacional de Desenvolvimento Científico e Tecnológico (CNPq \#503204/2011-0). P.C. Brum holds grants from 
Fundação de Amparo à Pesquisa do Estado de São Paulo (FAPESP \#2015/22814-5) and Conselho Nacional de Desenvolvimento Científico e Tecnológico

\section{References}

1. Brooks GA. Energy flux, lactate shuttling, mitochondrial dynamics, and hypoxia. Adv Exp Med Biol 2016; 903: 439-455, doi: 10.1007/978-1-4899-7678-9_29.

2. Brooks GA. Intra- and extra-cellular lactate shuttles. Med Sci Sports Exerc 2000; 32: 790-799, doi: 10.1097/00005768200004000-00011.

3. Chatham JC, Des Rosiers C, Forder JR. Evidence of separate pathways for lactate uptake and release by the perfused rat heart. Am J Physiol Endocrinol Metab 2001; 281: E794-E802, doi: 10.1152/ajpendo.2001.281.4.E794.

4. Philp A, Macdonald AL, Watt PW. Lactate--a signal coordinating cell and systemic function. J Exp Biol 2005; 208: 4561-4575, doi: 10.1242/jeb.01961.

5. Gabriel-Costa D, Cunha TF, Bechara LR, Fortunato RS, Bozi LH, Coelho Mde A, et al. Lactate up-regulates the expression of lactate oxidation complex-related genes in left ventricular cardiac tissue of rats. PLoS One 2015; 10: e0127843, doi: 10.1371/journal.pone.0127843.

6. Hashimoto T, Hussien R, Oommen S, Gohil K, Brooks GA. Lactate sensitive transcription factor network in L6 cells: activation of MCT1 and mitochondrial biogenesis. FASEB $J$ 2007; 21: 2602-2612, doi: 10.1096/fj.07-8174com.

7. Montoya JJ, Fernandez N, Monge L, Dieguez G, Villalon $\mathrm{AL}$. Nitric oxide-mediated relaxation to lactate of coronary circulation in the isolated perfused rat heart. $J$ Cardiovasc Pharmacol 2011; 58: 392-398, doi: 10.1097/FJC.0b013e $318226 \mathrm{bcf7}$.

8. Jaswal JS, Keung W, Wang W, Ussher JR, Lopaschuk GD. Targeting fatty acid and carbohydrate oxidation--a novel therapeutic intervention in the ischemic and failing heart. Biochim Biophys Acta 2011; 1813: 1333-1350, doi: 10.1016/ j.bbamcr.2011.01.015

9. Fukushima A, Milner K, Gupta A, Lopaschuk GD. Myocardial energy substrate metabolism in heart failure: from pathways to therapeutic targets. Curr Pharm Des 2015; 21: 3654 3664, doi: 10.2174/1381612821666150710150445.

10. Benjamin EJ, Blaha MJ, Chiuve SE, Cushman M, Das SR, Deo R, et al. Heart disease and stroke statistics-2017 update: a report from the American Heart Association. Circulation 2017; 135: e146-e603, doi: 10.1161/CIR.0000 000000000485.
(CNPq \#306261/2016-2). The funders had no role in study design, data collection and analysis, decision to publish, or preparation of the manuscript.

11. Go AS, Mozaffarian D, Roger VL, Benjamin EJ, Berry JD, Borden WB, et al. Executive summary: heart disease and stroke statistics - 2013 update: a report from the American Heart Association. Circulation 2013; 127: 143-152, doi: 10.1161/CIR.0b013e318282ab8f.

12. Rosenberg JC, Rush BF. An enzymatic-spectrophotometric determination of pyruvic and lactic acid in blood. Methodologic aspects. Clin Chem 1966; 12: 299-307.

13. Fortunato RS, Braga WM, Ortenzi VH, Rodrigues DC, Andrade BM, Miranda-Alves L, et al. Sexual dimorphism of thyroid reactive oxygen species production due to higher NADPH oxidase 4 expression in female thyroid glands. Thyroid 2013; 23: 111-119, doi: 10.1089/thy.2012.0142.

14. Veskoukis AS, Nikolaidis MG, Kyparos A, Kokkinos D, Nepka C, Barbanis S, et al. Effects of xanthine oxidase inhibition on oxidative stress and swimming performance in rats. Appl Physiol Nutr Metab 2008; 33: 1140-1154, doi: 10.1139/H08-102.

15. McCord JM, Fridovich I. Superoxide dismutase. An enzymic function for erythrocuprein (hemocuprein). J Biol Chem 1969; 244: 6049-6055.

16. Weydert CJ, Cullen JJ. Measurement of superoxide dismutase, catalase and glutathione peroxidase in cultured cells and tissue. Nat Protoc 2010; 5: 51-66, doi: 10.1038/nprot. 2009.197.

17. Bechara LR, Moreira JB, Jannig PR, Voltarelli VA, Dourado $\mathrm{PM}$, Vasconcelos AR, et al. NADPH oxidase hyperactivity induces plantaris atrophy in heart failure rats. Int $\mathrm{J}$ Cardiol 2014; 175: 499-507, doi: 10.1016/j.jjcard.2014.06.046.

18. Sirker A, Zhang M, Shah AM. NADPH oxidases in cardiovascular disease: insights from in vivo models and clinical studies. Basic Res Cardiol 2011; 106: 735-747, doi: 10.1007/ s00395-011-0190-z.

19. Murdoch $C E$, Zhang $M$, Cave AC, Shah AM. NADPH oxidase-dependent redox signalling in cardiac hypertrophy, remodelling and failure. Cardiovasc Res 2006; 71: 208-215, doi: 10.1016/j.cardiores.2006.03.016.

20. Opie LH. Coronary flow rate and perfusion pressure as determinants of mechanical function and oxidative metabolism of isolated perfused rat heart. $J$ Physiol 1965; 180: 529-541, doi: 10.1113/jphysiol.1965.sp007715. 